\title{
Biochemical and biophysical characterisation of immunoglobulin free light chains derived from an initially unbiased population of patients with light chain disease
}

\author{
Rebecca Sternke-Hoffmann ${ }^{1}$, Amelie Boquoi ${ }^{2}$, David Lopez ${ }^{2}$, Florian Platten ${ }^{3}$, Roland Fenk ${ }^{2}$, Rainer Haas ${ }^{\text {Corresp., }}$, \\ Alexander K Buell ${ }^{\text {Corresp. 1, } 4}$ \\ ${ }^{1}$ Institute of Physical Biology, Heinrich-Heine Universität Düsseldorf, Düsseldorf, Germany \\ 2 Department of Hematology, Oncology and Clinical Oncology, Heinrich-Heine Universität Düsseldorf, Düsseldorf, Germany \\ 3 Condensed Matter Physics Laboratory, Heinrich-Heine Universität Düsseldorf, Düsseldorf, Germany \\ 4 Department of Biotechnology and Biomedicine, Technical University of Denmark, Lyngby, Denmark \\ Corresponding Authors: Rainer Haas, Alexander K Buell \\ Email address: haas@med.uni-duesseldorf.de, alebu@dtu.dk
}

In light chain (LC) diseases, monoclonal immunoglobulin LCs are abundantly produced with the consequence in some cases to form deposits of a fibrillar or amorphous nature affecting various organs, such as heart and kidney. The factors that determine the solubility of any given LC in vivo are still not well understood. We hypothesize that some of the biochemical properties of the LCs that have been shown to correlate with amyloid fibril formation in patients also can be used as predictors for the degree of kidney damage in a patient group that is only biased by protein availability. We performed detailed biochemical and biophysical investigations of light chains extracted and purified from the urine of a group of 20 patients with light chain disease. For all samples that contained a sufficiently high concentration of LC, we quantified the unfolding temperature of the LCS, the monomer-dimer distribution, the digestibility by trypsin and the formation of amyloid fibrils under various conditions of $\mathrm{pH}$ and reducing agent. We correlated the results of our biophysical and biochemical experiments with the degree of kidney damage in the patient group and found that most of these parameters do not correlate with kidney damage as defined by clinical parameters. However, the patients with the greatest impairment of kidney function have light chains which display very poor digestibility by trypsin. Most of the LC properties reported before to be predictors of amyloid formation cannot be used to assess the degree of kidney damage. Our finding that poor trypsin digestibility correlates with kidney damage warrants further investigation in order to probe a putative mechanistic link between these factors. 


\section{Biochemical and biophysical characterisation of}

2 immunoglobulin free light chains derived from an

3 initially unbiased population of patients with light

4 chain disease

5

6

7

8

9

10

11

12

13

Rebecca Sternke-Hoffmann 1 , Amelie Boquoi², David Lopez ${ }^{2}$, Florian Platten ${ }^{3}$, Roland Fenk ${ }^{2}$, Rainer Haas² and Alexander K. Buell ${ }^{1,4}$

${ }^{1}$ Institute of Physical Biology, Heinrich-Heine-University Düsseldorf, Germany

${ }^{2}$ Department of Hematology, Oncology and Clinical Oncology, Heinrich-Heine-University Düsseldorf, Germany

${ }^{3}$ Condensed Matter Physics Laboratory, Heinrich-Heine-University Düsseldorf, Germany

${ }^{4}$ Department of Biotechnology and Biomedicine, Technical University of Denmark, Lyngby, Denmark

Corresponding Authors:

Alexander Buell ${ }^{1,4}$

Søltofts Plads, Building 227, 2800 Kgs Lyngby, Denmark

Email address: alebu@dtu.dk;

Rainer Haas ${ }^{2}$

Moorenstraße 5, 40225 Düsseldorf, Germany

Email address: haas@med.uni-duesseldorf.de

\section{Abstract}

In light chain (LC) diseases, monoclonal immunoglobulin LCs are abundantly produced with the consequence in some cases to form deposits of a fibrillar or amorphous nature affecting various organs, such as heart and kidney. The factors that determine the solubility of any given LC in vivo are still not well understood. We hypothesize that some of the biochemical properties of the LCs that have been shown to correlate with amyloid fibril formation in patients also can be used as predictors for the degree of kidney damage in a patient group that is only biased by protein availability.

We performed detailed biochemical and biophysical investigations of light chains extracted and purified from the urine of a group of 20 patients with light chain disease. For all samples that contained a sufficiently high concentration of LC, we quantified the unfolding temperature of the 
39 LCs, the monomer-dimer distribution, the digestibility by trypsin and the formation of amyloid

40 fibrils under various conditions of $\mathrm{pH}$ and reducing agent.

41 We correlated the results of our biophysical and biochemical experiments with the degree of

42 kidney damage in the patient group and found that most of these parameters do not correlate with

43 kidney damage as defined by clinical parameters. However, the patients with the greatest

44 impairment of kidney function have light chains which display very poor digestibility by trypsin.

45 Most of the LC properties reported before to be predictors of amyloid formation cannot be used

46 to assess the degree of kidney damage. Our finding that poor trypsin digestibility correlates with

47 kidney damage warrants further investigation in order to probe a putative mechanistic link

48 between these factors.

\section{Introduction}

51 The formation of insoluble aggregates by proteins can be associated with a broad range of human

52

53

54

55

56

57

58

59

60

61

62

63

64

65

66

67

68

69

70

71

72

73

74

75

76

77

78 disorders, many of which are neurodegenerative in nature (1-3). Protein deposition in other organs than the central nervous system can also lead to a wide variety of diseases. In the case of the deposition of ordered fibrillar aggregates (amyloid fibrils) affecting various organs the related disorders are known as systemic amyloidoses (4).

A particular class of such protein deposition disorders is represented by the light chain diseases, which are characterized by the occurrence of monoclonal free light chains in blood and urine (5). In general, the monoclonal free light chains of either kappa or lambda isotype are the secreted product of monoclonal plasma cells residing in the bone marrow. Dependent on the type of the underlying B-cell disorder, they may reflect a monoclonal gammopathy of uncertain significance (MGUS), a smoldering or full blown multiple myeloma (MM) with exclusive (Bence Jones MM) or substantial light chain secretion in addition to the complete immunoglobulin. Each light chain protein has a unique amino acid sequence which is determined by somatic recombination and various mutations (6). The amino acid sequence, together with potential post-translational modifications and in interplay with the local conditions in the organism, such as local $\mathrm{pH}$ or presence of proteases, determines the in vivo behavior of the light chain. The sequence diversity translates into a diverse clinical picture, as far as organ involvement and severity of organ damage are concerned (7). Aggregation of light chains and fragments of low solubility can lead to different diseases, such as light chain deposition disease (LCDD), where the LC forms amorphous aggregates $(8,9)$, and the AL-amyloidosis, where the LC forms amyloid fibrils (10). At present, it is not possible to decide upon first diagnosis of a patient with light chain disease, whether the particular monoclonal light chain found in their blood at increased concentration is prone to form amyloid fibrils or amorphous deposits, or remain soluble and get excreted quantitatively through the urine. Even if the sequence of the particular light chain is known, its solubility inside the organism cannot currently be predicted. Therefore, there is a need both for increased mechanistic understanding of LC deposition and for easy, rapid and reliable diagnostic procedures that are able to assess the potential of a given light chain to cause damage through deposition. 
79 Much work has been carried out in recent years on the study of the biophysical properties of LCs

80 and to correlate the results with, in particular, their amyloid fibril formation in vivo (11-15).

81 Most of these studies have relied on the a priori knowledge that a given light chain forms, or not,

82 amyloid fibrils in vivo. In this study, we set out to test the hypothesis that (some of) the

83 biochemical and biophysical properties of light chains previously reported specifically in the

84 context of amyloidosis also correlate with the severity of general light chain disease symptoms,

85 in particular impairment of kidney function. In our study, we examined 20 patients presenting

86 with light chains in their urine. Most of them had multiple myeloma with a large variety of

87 symptoms. For our in vitro assessment, we isolated protein from the urine of these patients,

88 where the proteins appear in high concentration, when the production is significantly increased

89 and the ability to reabsorb the filtered proteins is exceeded, and characterized the samples in

90 detail with biochemical and biophysical methods. We did not apply any particular selection

91 criteria to include patients into the study, but excluded those samples that did not contain a

92 sufficient concentration of light chains.

93

94

95

96

97

98

99

100

101

102

103

104

105

106

107

108

109

110

111

112

113

114

115

116

117

118

\section{Materials \& Methods}

\section{Patients}

The study described in this manuscript has been reviewed and approved by the ethics committee of the university hospital Düsseldorf and all patients of whom samples were used in the study have signed an informed consent (study number 5926R and registration ID 20170664320). The in vitro studies were performed using protein isolated from urine samples of 20 patients (6 females, 14 males, median age 61.5 years with a range between 45 and 76 years) with multiple myeloma of various subtypes and one patient with Amyloidosis as detailed in Table 1 (patient characteristics). For the majority of patients - P008 and 009 had a cast nephropathy - a histopathological examination of the kidney was not available since the corresponding invasive diagnostic procedure was not necessary for the therapy decision-making process as they were diagnosed according to IMWG criteria. Thus, there was no initial bias in the selection of patient, as the common denominator for inclusion into the study was solely the presence of a monoclonal light chain in the peripheral blood and in the urine, while the type of ligt chain disease (MM vs. Amyloidosis) did not play a role.

As far as the type of light chain is concerned, 14 patients with Kappa and 6 patients with Lambda type chain were part of the study. There was a large variation with regard to the light chain concentration in the serum, with concentrations between $2.2 \mathrm{mg} / \mathrm{l}$ and $11,000 \mathrm{mg} / \mathrm{l}$. Two of our patients presented with renal insufficiency and required dialysis, while four of them had no signs of functional renal impairment. We divided the patients into three different groups (I,II,III) according to their chronic kidney disease (CKD)-stage (1-5) at the time of diagnosis. Groups I corresponds to stages 1 and 2 , group II to stage 3 and group III to stages 4 and 5 . At the time when the urine samples were collected, 17 patients were diagnosed de novo, whereas three patients with multiple myeloma had received induction therapy with bortezomib, 
119 cyclophosphamide and dexamethasone. The duration of their disease at the time of the

120 examination was therefore relatively short with a median of 6 weeks varying from 4 to 12 weeks.

121

122

\section{Experimental Methods}

123

Detailed descriptions of the methods are provided in the supplemental materials.

124

Sample preparation

125

The protein content of a $24 \mathrm{~h}$ urine collection was precipitated by ammonium sulfate ( $70 \%$

126 saturation) and the light chains were purified by size-exclusion chromatography on an ÄKTA

127 pure chromatography system (GE Healthcare) using a Superdex 75 10/300 GL column.

Analysis of the LC and HSA-ratio

129

The purity and LC content of the samples was evaluated based on a combination of SDS-PAGE

130 gels and Western blots with antibodies against human kappa light chain, human lambda light

131 chain or human serum albumin (HSA).

132

133

Differential scanning calorimetry (DSC)

134

Thermal unfolding of the various light chains was studied using a MicroCal VP-DSC instrument (Malvern, UK) by performing temperature ramps on the $\mathrm{LC}$ solutions from $10^{\circ} \mathrm{C}$ to $90^{\circ} \mathrm{C}$ with a heating rate of $0.8^{\circ} \mathrm{C} / \mathrm{min}$. The degree of refolding was estimated by the ratio of the areas under the unfolding peaks of the second to the first temperature scan.

138

\section{Determination of the dimer content}

140

To determine the ratio of dimers to monomers of the various LC's, the proteins were run, before purification via SEC, on denaturing, non-reducing SDS-PAGE gels, which were stained using

142 Coomassie blue.

143

144

Proteolysis

146

LCs at an estimated concentration of $33 \mu \mathrm{M}$ were incubated with bovine trypsin (molar ratio $1: 100)$ at $37^{\circ} \mathrm{C}$ in $10 \mathrm{mM}$ phosphate buffer, $\mathrm{pH} 7.5$ with $1 \mathrm{M}$ urea, . The preparation was done on ice and the aliquot for the first time point was collected after trypsin addition and immediately inhibited by adding trypsin inhibitor (at an excess of 2:1). Further aliquots were taken 1,2, 18, 24 and $48 \mathrm{~h}$ after the addition of the trypsin and quenched by the addition of trypsin inhibitor.

151 The samples were analyzed by SDS-PAGE.

Amyloid fibril formation in vitro

154 Different solution conditions ( $\mathrm{pH}$, trypsin) were tested for their potential to induce amyloid fibril $\mu \mathrm{M}$ of the fluorescent dye Thioflavin-T (ThT) in a multi-well plate reader.

\section{Results \& Discussion}


159

160

161

162

163

164

165

166

167

168

169

170

171

172

173

174

175

176

177

178

179

180

181

182

183

184

185

186

187

188

189

190

191

192

193

194

195

196

197

198

\section{Clinical data}

The results presented are based on the urine samples of 20 patients with a monoclonal light chain (14 Kappa and 6 Lambda) related to different types of multiple myeloma and one patient with confirmed amyloidosis. Common denominator for inclusion into this in vitro study was the availability of a sufficient amount and purity of protein extractable from the urine. Further experiments were then performed with those samples that contained a dominant proportion of LCs. As a consequence, two samples (P008 and P009) were excluded from further analysis, as they mainly contained HSA. The data for the evaluation of LC content in the samples can be found in the Supplementary Materials (Figure S1).

We have chosen this clinically unbiased approach (i.e. no exclusion of patients based on clinical phenotype) in terms of day-to-day practicability rather than applying restrictive inclusion criteria. By doing so, we expected a better evaluation of the utility and relevance of the biochemical and biophysical methods within the diagnostic work flow At the time of inclusion into the study, the majority of patients were newly diagnosed without previous therapy. Two of the patients required dialysis. By investigating a potential relationship between the LC concentration in the blood and the degree of renal impairment at the time of the assessment we found no statistically relevant relationship (correlation coefficient of 0.16 with p-value of 0.54 ).

We were also interested in the further course of the disease and examined the kidney looking for an improvement of the creatinine concentration in serum during the course of the treatment. Without adequate information on $\mathrm{P} 005$ and $\mathrm{P} 013$ it was interesting to note, that of the 18 patients available for assessment even patients of the category 3 showed an improved kidney function after treatment. On the other hand, the renal insufficiencies of P002 and P003 are probably irreversible. The kidney function of most of the patients of category 1 did not change significantly, as their kidney function at the time of examination was not or only moderately impaired. Previous studies have shown that patients with LCDD and active MM displayed a greater improvement of kidney function in comparison to pure LCDD (16).

\section{Thermal stability}

It has been proposed that the thermodynamic stability of a light chain correlates with its tendency to form amyloid fibrils (12,17-20), but this correlation has not been observed in all studies (21). Here we tested whether the stability against thermal unfolding correlated with the severity of the disease symptoms, independently of the fact if amyloid fibrils or other types of aggregates cause those symptoms. The thermal stability of the proteins was investigated using differential scanning calorimetry (DSC). The transition temperatures $\left(\mathrm{T}_{\mathrm{m}}\right)$ of the different samples range from $50^{\circ} \mathrm{C}$ to nearly $70^{\circ} \mathrm{C}$ (Figure $1 \mathrm{~b}$ ). In contrast to the other samples, which displayed one distinct peak for the unfolding of the LC, the thermogram of $\mathrm{P} 004$ showed two peaks; hence the presented $\mathrm{T}_{\mathrm{m}} 57.7^{\circ} \mathrm{C}$ is a mean of the $\mathrm{T}_{\mathrm{m}}$ values of both peaks $\left(53.2^{\circ} \mathrm{C}\right.$ and $\left.62.1^{\circ} \mathrm{C}\right)$. The mean unfolding temperature of the kappa LCs of this study is $53.7^{\circ} \mathrm{C}$ and the average unfolding temperature of the lambda LCs is $58.6^{\circ} \mathrm{C}$. Lambda $\mathrm{LC}$ have on average a greater 
199

200

201

202

203

204

205

206

207

208

209

210

211

212

213

214

215

216

217

218

219

220

221

222

223

224

225

226

227

228

229

230

231

232

233

234

235

236

237

238

stability against thermal unfolding, which is in agreement with published reports (21). No correlation was observed between the thermal stability and the severity of the patient's kidney function.

It has been reported that LCs derived from MM-patients, in contrast to those from AL-patients, were not able to refold after heat denaturation (13). Therefore it has been speculated that this experimental parameter could be predictive for the in vivo behavior. In order to investigate the degree of reversibility of the thermal unfolding, the samples were heated for a second time, following a first ramping from $10^{\circ} \mathrm{C}$ to $90^{\circ} \mathrm{C}$ and subsequent cooling down within the DSC liquid cell. If the protein can quantitatively refold into the native conformation after thermal unfolding, the thermograms of the two successive scans should not differ significantly. If only a fraction of the protein can refold into the initial structure, and the remaining fraction misfolds or aggregates, the integral of the DSC thermogram, i.e. the enthalpy of unfolding, correspondingly decreases or even vanishes. This decrease in the apparent enthalpy of unfolding is caused by the fact that only a correctly folded protein has a well-defined unfolding transition, whereas

misfolded or aggregated protein comprises a multitude of states, often highly stable, that do not undergo a thermally induced transition at a well-defined temperature.

The observed degree of refolding upon reheating up to $90^{\circ} \mathrm{C}$ was highly variable. Some LCs did not display a peak in the thermogram at the second temperature ramp, whereas other examined LCs show a high reversibility (up to 70\%) of folding (Figure $1 \mathrm{c}$ ). Again, we found no apparent correlation between the ability of refolding after heating to $90^{\circ} \mathrm{C}$ and the patients' renal function. Although of more fundamental interest, we also tested for a correlation between thermal stability and refolding ability of the LCs (Figure S5) and found a weak inverse correlation, i.e. the more thermostable a given LC, the smaller its ability to refold. We discuss possible origins of this connection in the SI.

\section{Dimerization}

The conformation of pathogenic LC proteins is typically dimeric $(21,22)$, and it has been proposed that the tendency to dimerize can be used as a diagnostic parameter for the tendency to form amyloid fibrils, whereby light chains in serum samples derived from AL patients show abnormally high levels of monoclonal free light chain (FLC) dimers on a western blot (23). On the other hand, the formation of dimers seems to prevent the LC to aggregate in vitro. The formation of amyloid fibrils was observed when the dimer was destabilized and dissociated into monomers (24-26).

In order to test whether the degree of dimerization can be used as a predictor for general disease severity in the form of kidney impairment in LC disease, we performed similar experiments in our study. The relative fraction of dimers of the LCs after precipitating the proteins from the urine, redissolving them in buffer and purification by dialysis was determined with a nonreducing SDS-PAGE gel and quantified photometrically (Figure 2). The fraction of dimers is high in some of the LC, and none of the examined LCs occurs exclusively in its monomeric form. A clear correlation between the fraction of dimerized LC and the renal function of the 
239

240

241

242

243

244

245

246

247

248

249

250

251

252

253

254

255

256

257

258

259

260

261

262

263

264

265

266

267

268

269

270

271

272

273

274

275

276

277

278

patients was not observed. In the case of P011, the sample from the only patient with confirmed amyloidosis, the dimer fraction of 0.75 is in accordance with the findings of the FLC-MDPA test that amyloid prone LCs occur mainly in their dimeric forms (23).

\section{Trypsin digestion}

The structural flexibility and dynamics of the LC have been proposed to be correlated with the ability to form amyloid fibrils in vivo. LC derived from AL-patients displayed rapid proteolysis with trypsin under mildly destabilizing conditions which suggests increased dynamics of the native fold of the amyloid-forming LC (27). These structural dynamics may be required to form amyloid fibrils under physiological conditions. In the present study, we performed such experiments in order to test whether structural flexibility of the light chains correlates with the severity of LC disease symptoms.

The structural flexibility and dynamics of the LC were probed by digesting the proteins using trypsin in the presence of $1 \mathrm{M}$ urea. Fast proteolysis under these conditions may suggest increased dynamics in the native fold of the LC, which allows the protease to access the cleavage sites more easily. Of course it is important to remember that the sequence variability between different light chains will lead to different numbers and accessibilities of trypsin cleavage sites. The availability of sequence information of the investigated light chains would therefore be beneficial for the detailed analysis of their proteolytic susceptibility, and will be included in a future study. The digestion time course was analyzed using SDS-PAGE gels by determining how much native protein (monomer and dimer combined) was still present after $48 \mathrm{~h}$ incubation with trypsin (1:100 molar ratio) at $37^{\circ} \mathrm{C}$ (Figure $\left.3 \mathrm{a}, \mathrm{b}\right)$.

In addition to correlating trypsin digestion with kidney impairment, we also correlated it with the thermal stability of the LCs (Figure S5). A weak inverse correlation is observed, i.e. the more thermostable a LC, the less it is susceptible to be degraded by trypsin. While this connection is also of interest in a more fundamental protein science context, it does lend some support to the hypothsis underlying the digestion assay, see discussion in the SI.

These proteolysis experiments were in addition also conducted inside a multi-well plate reader in the presence of the fluorescent, amyloid specific dye ThT. Only seven of the tested samples showed an increase in fluorescence intensity indicating amyloid fibril formation (Figure $3 \mathrm{~d}$ and Figure S2). Six of these 7 amyloid positive LCs were almost completely digested after $48 \mathrm{~h}$, suggesting that proteolytic cleavage of the LC facilitates the formation of amyloid fibrils. Proteolysis-induced amyloid fibril formation of light chains is a well-established phenomenon, and even the aggregation of non-amyloidogenic light chains after acidic proteolysis was observed $(28,29)$. The presence of amyloid fibrils was confirmed by atomic force microscopy (AFM, Figure 3 e). Interestingly, the samples of patients of category three, i.e. the patients most severely affected by impairment of kidney function, were among the least digestible proteins tested. This observed behavior should be compared to the findings on the behavior of amyloid prone LCs previously reported (27), whereby it was found that amyloidgenic LCs are most easily digested. Interestingly, the only established amyloidosis-related LC of our dataset (P011) was not 
279 digested during the investigated time period and was also not observed to form amyloid fibrils

280 under the conditions of this experiment. However, the presence, in our data set, of only a single

281 LC known to form amyloid fibrils in vivo did not allow us to test the previously reported

282 correlation between proteolytic degradability and amyloid fibril formation.

283 On the other hand, while easy degradability might increase the risk for amyloid formation, our

284 data set does suggest that LCs which are difficult to proteolytically degrade tend to be associated

285 with impairment of the renal function. A putative mechanism by which this association can be

286 explained is the more rapid accumulation, probably in the form of amorphous aggregates, of non-

287 cleavable LC in the kidneys. At the same time, it has to be kept in mind that the proteases

288 responsible for the degradation of LCs in vivo have cleavage patterns distinct from that of trypsin

289 and therefore the digestibility by trypsin will only have limited predictive power for the

290 digestibility of a given LC in vivo.

291

292

293

294

295

296

297

298

299

300

301

302

303

304

305

306

307

308

309

310

311

312

313

314

315

316

317

318

\section{$\mathrm{pH}$-dependent amyloid formation}

Given our findings that digestion by trypsin facilitated amyloid fibril formation of our patientderived LCs, we further tested whether variations in solution conditions ( $\mathrm{pH}$, reducing agent) are also able to induce amyloid fibril formation in a subset of our samples. We found that at neutral $\mathrm{pH}$ in the presence of the reducing agent TCEP at $37^{\circ} \mathrm{C}$ almost all the samples (except P002, P013, P014, P016, P020) show an increase in ThT fluorescence over time, suggestive of amyloid fibril formation, (see Figure 4 a for representative data and Figure $\mathrm{S} 4$ for an overview). It is known that a reduction of mostly intermolecular disulfide bridges of light chains can induce amyloid formation (21). If the $\mathrm{pH}$ was lowered towards more acidic values $(\sim \mathrm{pH} 4)$,

fragmentation of the chains was observed, and in most cases amyloid fibril formation was absent at acidic $\mathrm{pH}$ in the presence of TCEP. Presumably, the degree of fragmentation of the LC through the combined effects of TCEP and low $\mathrm{pH}$ was too strong, such that the resulting short fragments were unable to form amyloid fibrils.

On the other hand, no amyloid fibril formation was observed at neutral $\mathrm{pH}$ in the absence of a reducing agent (see Figure $4 \mathrm{~b}$ ). If, however, in the absence of reducing agent the $\mathrm{pH}$ was decreased, some of the LCs showed formation of amyloid fibrils at $\mathrm{pH} 4$, in particular also P011, derived from the patient with confirmed amyloidosis (Figure S3). Low pH destabilizes the proteins and mildly destabilizing conditions are known to accelerate aggregation (30). For example, investigations of the amyloidogenic variable domain SMA showed relatively nativelike intermediates, but with significant changes of the tertiary structure, at $\mathrm{pH} 4$ (31). A summary of the aggregation behavior at $\mathrm{pH} 4$, together with the corresponding patient categories of the samples, is given in figure $4 \mathrm{f}$ ). Seven LCs formed amyloid fibrils at $\mathrm{pH} 4$. Figure $4 \mathrm{c}$ ) displays a representative example of the observed aggregation kinetics of sample $\mathrm{P} 005 \mathrm{at} \mathrm{pH} 4$. The resulting amyloid fibrils were also in all cases examined using AFM imaging (see Figure $4 \mathrm{~d}$ and e) for a representative example, and Figure S3). The fibrils seem to form clusters/higher order assemblies at this $\mathrm{pH}$, but also individual fibrils were detected. Some of the LCs displayed amyloid fibril formation only at $\mathrm{pH} 4$ and did not form amyloid fibrils if the $\mathrm{pH}$ was decreased to 
$319 \mathrm{pH} 3$ and below. Very low $\mathrm{pH}$ may lead to too substantial fragmentation of the LC (32), similar 320 to the combined effect of reducing agent and less acidic $\mathrm{pH}$ discussed above. There is no obvious 321 correlation observable between the impairment of kidney function at the time of diagnosis, i.e.

322 the patient category, and the aggregation behavior under the investigated solution conditions.

323

324

325

326

327

328

329

330

331

332

333

334

335

336

337

338

339

340

341

342

343

344

345

346

347

348

349

350

351

352

353

354

355

356

357

\section{Conclusions}

This study provides a comprehensive evaluation of the in vitro biophysical and biochemical behavior of patient-derived monoclonal light chains. The majority of patients had been diagnosed de novo with multiple myeloma, while a histopathological examination was performed in 3 patients leading to the diagnosis of cast nephropathy in 2 patients and AL-Amyloidosis in one. We used an unbiased group of patients as they came to our hospital during our recruitment phase. Therefore, different from recent studies reporting on patients with light chain disease we cannot make an a priori separation into two distinct groups on the basis of the amyloid formation behavior of the respective LC proteins. However, an a posteriori bias was introduced into our patient group by excluding two samples from further analysis, because they did not contain a sufficient concentration of light chains, but were dominated by albumin.

Starting with the thermal stability of the LCs and their ability to refold after thermal unfolding we could not find a clear correlation with the degree of kidney damage (see Figure 5 for correlation coefficients). Also, the relative amount of dimer and the aggregation behavior at $\mathrm{pH} 4$ were not correlated to the different patient categories. The digestibility by trypsin of the LCs yielded no clear correlation with kidney damage for our samples. According to published results (27), LCs derived from AL-amyloidosis patients could be relatively easily digested whereas LCs from patients with Multiple Myeloma seemed to be more resistant against digestion by trypsin. Trypsin digestion is here used as a proxy for structural dynamics, with the caveat that different LCs are likely to differ in the number and availability of trypsin cleavage sites.

The most noteworthy result of our study is that in our dataset, the LCs of the three patients with the greatest degree of renal impairment were only digested by trypsin to a very small extent. Therefore, while easily digestible LC may have a tendency to form toxic amyloid fibrils in vivo, our results suggest that indigestible LCs may nevertheless be able to induce severe kidney damage, due to their overall higher structural stability their resulting ability to accumulate in the kidneys. This conclusion will, however, need to remain somewhat speculative until sequence information of the LCs of this study becomes available (ongoing work), which will allow to investigate how physiologically more relevant proteases can digest these LCs.

The particular feature of LC diseases, namely that every patient displays a LC of a unique amino acid sequence, makes it necessary to substantially enlarge the currently available data set that links biophysical, biochemical and sequence information with clinical disease symptoms and the present study aims to contribute to this task. In order to obtain a better mechanistic understanding of the in vivo behavior of the LCs, a more detailed biophysical investigation, as well as sequence 
358

359

360

361

362

363

364

365

366

367

368

369

370

371

372

373

374

375

376

377

378

379

380

381

382

383

384

385

386

387

388

389

390

391

392

393

394

395

determinations (see above) of the extracted light chains, are required. These are the subject of ongoing studies of our groups.

\section{References}

1. Dobson CM. Protein folding and misfolding. Nature 2003;52:884-90.

2. Westermark P, Benson MD, Buxbaum JN, Cohen AS, Frangione B, Ikeda S-I, Masters CL, Merlini G, Saraiva MJ, Sipe JD. Amyloid: toward terminology clarification. Report from the Nomenclature Committee of the International Society of Amyloidosis. Amyloid 2005;12:1-4.

3. Knowles TPJ, Vendruscolo M, Dobson CM. The amyloid state and its association with protein misfolding diseases. Nat Rev Mol Cell Biol 2014;15:384-96.

4. Wechalekar AD, Gillmore JD, Hawkins PN. Systemic amyloidosis. Lancet 2016;387:264154.

5. Edelman GM, Gally JA. The nature of Bence-Jones proteins. Chemical similarities to

polypetide chains of myeloma globulins and normal gamma-globulins. J Exp Med 1962;116:207-27.

6. Sakano H, Hüppi K, Heinrich G, Tonegawa S. Sequences at the somatic recombination sites of immunoglobulin light-chain genes. Nature 1979;280:288-94.

7. Enqvist S, Sletten K, Stevens FJ, Hellman U, Westermark P. Germ Line Origin and Somatic

Mutations Determine the Target Tissues in Systemic AL-Amyloidosis. PLoS One ;2:e981.

8. Sanders PW, Booker BB. Pathobiology of cast nephropathy from human Bence Jones proteins. J Clin Invest 1992;89:630-9.

9. Buxbaum J, Gallo G. Nonamyloidotic monoclonal immunoglobulin deposition disease. Lightchain, heavy-chain, and light- and heavy-chain deposition diseases. Hematol Oncol Clin North Am 1999;13:1235-48.

10. Glenner GG, Ein D, Terry WD. The immunoglobulin origin of amyloid. Am J Med 1972;52:141-7.

11. Arosio P, Owczarz M, Müller-Späth T, Rognoni P, Beeg M, Wu H, Salmona M, Morbidelli

M. In vitro aggregation behavior of a non-amyloidogenic $\lambda$ light chain dimer deriving from U266 multiple myeloma cells. PLoS ONE 2012;7:e33372.

12. Blancas-Mejía LM, Horn TJ, Marin-Argany M, Auton M, Tischer A, Ramirez-Alvarado M. Thermodynamic and fibril formation studies of full length immunoglobulin light chain AL-09 and its germline protein using scan rate dependent thermal unfolding. Biophys Chem 2015;207:13-20.

13. Andrich K, Hegenbart U, Kimmich C, Kedia N, Bergen HR, Schönland S, Wanker E, Bieschke J. Aggregation of Full-length Immunoglobulin Light Chains from Systemic Light Chain Amyloidosis (AL) Patients Is Remodeled by Epigallocatechin-3-gallate. J Biol Chem 2017;292:2328-44. 
396

397

398

399

400

401

402

403

404

405

406

407

408

409

410

411

412

413

414

415

416

417

418

419

420

421

422

423

424

425

426

427

428

429

430

431

432

433

434

435

14. Brumshtein B, Esswein SR, Sawaya MR, Rosenberg G, Ly AT, Landau M, Eisenberg DS. Identification of two principal amyloid-driving segments in variable domains of Ig light chains in systemic light-chain amyloidosis. J Biol Chem 2018;293:19659-71.

15. Weber B, Hora M, Kazman P, Göbl C, Camilloni C, Reif B, Buchner J. The Antibody LightChain Linker Regulates Domain Orientation and Amyloidogenicity. J Mol Biol 2018;430:492540.

16. Muzaffar J, Khan AM, Unnikrishnan A, Narayan P, Wakefield D, Moreb JS. Characteristics of Light Chain Deposition Disease (LCDD) and Factors Affecting Outcome after Treatment. Blood 2017;130:5513-5513.

17. Hurle MR, Helms LR, Li L, Chan W, Wetzel R. A role for destabilizing amino acid replacements in light-chain amyloidosis. Proc Natl Acad Sci USA 1994;91:5446-50.

18. Klimtchuk ES, Gursky O, Patel RS, Laporte KL, Connors LH, Skinner M, Seldin DC. The critical role of the constant region in thermal stability and aggregation of amyloidogenic immunoglobulin light chain. Biochemistry 2010;49:9848-57.

19. Raffen R, Dieckman LJ, Szpunar M, Wunschl C, Pokkuluri PR, Dave P, Wilkins SP, Cai X, Schiffer M, Stevens FJ. Physicochemical consequences of amino acid variations that contribute to fibril formation by immunoglobulin light chains. Protein Sci 1999;8:509-17.

20. del Pozo Yauner L, Ortiz E, Sánchez R, Sánchez-López R, Güereca L, Murphy CL, Allen A, Wall JS, Fernandez-Velasco DA, Solomon A, Becerril B. Influence of the germline sequence on the thermodynamic stability and fibrillogenicity of human lambda 6 light chains. Proteins 2008;72:684-92.

21. Bernier GM, Putnam FW. Monomer - Dimer forms of Bence Jones Proteins. Nature 1963;200:223-5.

22. Epp O, Lattman EE, Schiffer M, Huber R, Palm W. The molecular structure of a dimer composed of the variable portions of the Bence-Jones protein REI refined at 2.0-A resolution. Biochemistry 1975;14:4943-52.

23. Gatt ME, Kaplan B, Yogev D, Slyusarevsky E, Pogrebijski G, Golderman S, Kukui O, Livneh A. The use of serum free light chain dimerization patterns assist in the diagnosis of AL amyloidosis. Br J Haematol 2018;182:86-92.

24. Brumshtein B, Esswein SR, Landau M, Ryan CM, Whitelegge JP, Phillips ML, Cascio D, Sawaya MR, Eisenberg DS. Formation of amyloid fibers by monomeric light chain variable domains. J Biol Chem 2014;289:27513-25.

25 . Wolwertz ML, Nguyen PT, Quittot N, Bourgault S. Probing the role of $\lambda 6$ immunoglobulin light chain dimerization in amyloid formation. Biochim Biophys Acta 2016;1864:409-18.

26. Nawata M, Tsutsumi H, Kobayashi Y, Unzai S, Mine S, Nakamura T, Uegaki K, Kamikubo H, Kataoka M, Hamada D. Heat-induced native dimerization prevents amyloid formation by variable domain from immunoglobulin light-chain REI. FEBS J 2017;284:3114-27.

27. Oberti L, Rognoni P, Barbiroli A, Lavatelli F, Russo R, Maritan M, Palladini G, Bolognesi M, Merlini G, Ricagno S. Concurrent structural and biophysical traits link with immunoglobulin light chains amyloid propensity. Sci Rep 2017;7:16809.

Peer) reviewing PDF | (2019:10:41883:1:1:NEW 21 Jan 2020) 
436 28. Linke RP, Zucker-Franklin D, Franklin EC. Morphologic, Chemical, and Immunologic 437 Studies of Amyloid-Like Fibrils Formed from Bence Jones Proteins by Proteolysis. J Immunol 438 1973;111:10-23.

439 29. Glenner GG, Ein D, Eanes ED, Bladen HA, Terry W, Page DL. Creation of “amyloid” fibrils 440 from Bence Jones proteins in vitro. Science 1971;174:712-4.

441 30. Hu D, Qin Z, Xue B, Fink AL, Uversky VN. Effect of Methionine Oxidation on the 442 Structural Properties, Conformational Stability, and Aggregation of Immunoglobulin Light 443 Chain LEN. Biochemistry 2008;47:8665-77.

444 31. Khurana R, Gillespie JR, Talapatra A, Minert LJ, Ionescu-Zanetti C, Millett I, Fink AL. 445 Partially Folded Intermediates as Critical Precursors of Light Chain Amyloid Fibrils and 446 Amorphous Aggregates. Biochemistry 2001;40:3525-35.

447 32. Vlasak J, Ionescu R. Fragmentation of monoclonal antibodies. MAbs 2011;3:253-63. 


\section{Figure 1}

Thermal stability and reversibility of unfolding.

(A) Thermograms of the first and second DSC scans of P001 and P006. (B) Unfolding temperatures determined from differential scanning calorimetry (DSC) experiments of the different samples allocated in the three patient categories according to renal impairment (CDK stage): I (green), II (blue), III (red) (left). (C) The fraction of native protein that unfolds during the second scan. The boxes range between 25 and 75 percent, the median is visualized by the horizontal line and the mean by the small square. P008, P009 (Iow LC content), P014 and P018 (low sample availability) are excluded from this analysis. 

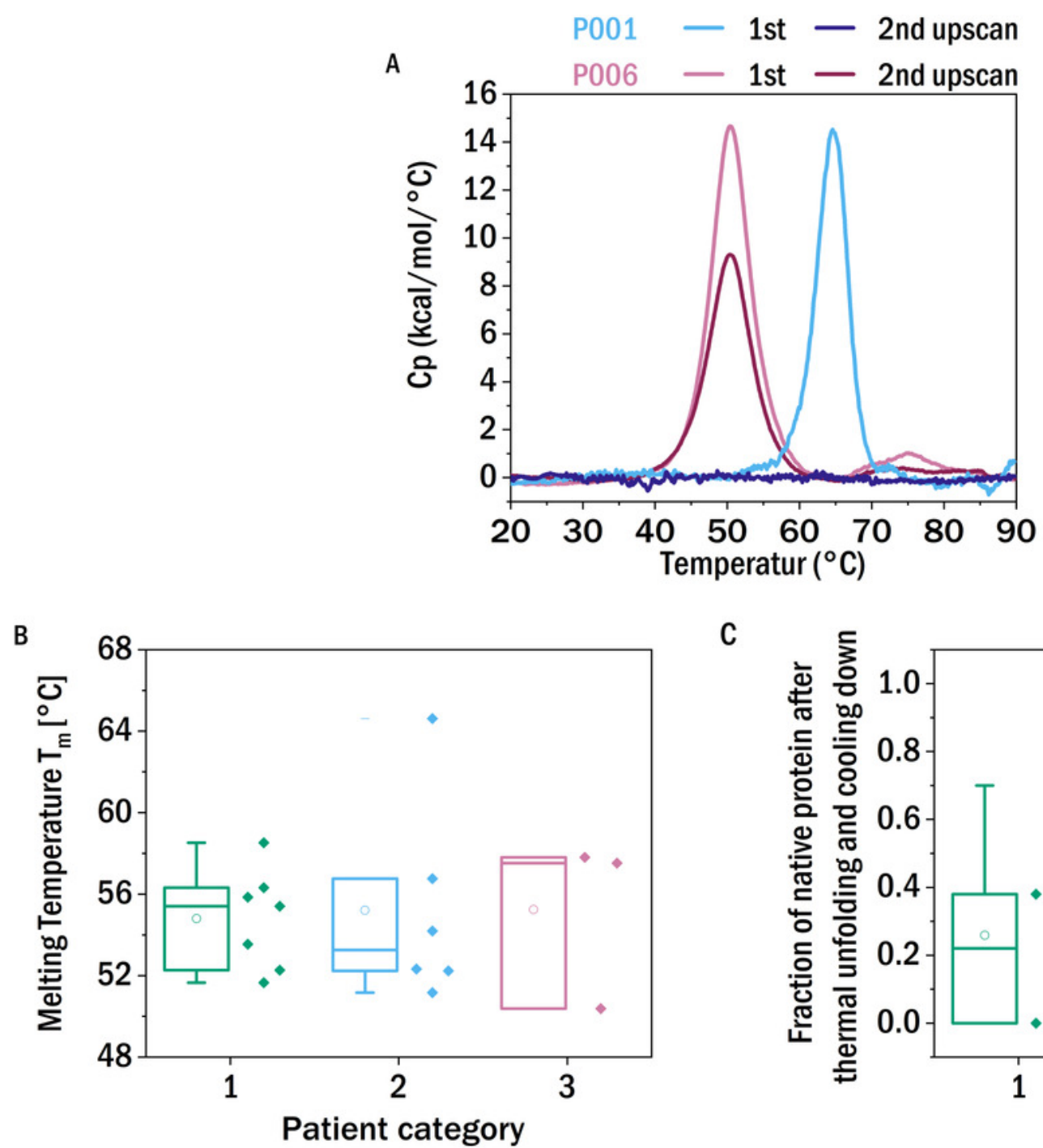

C

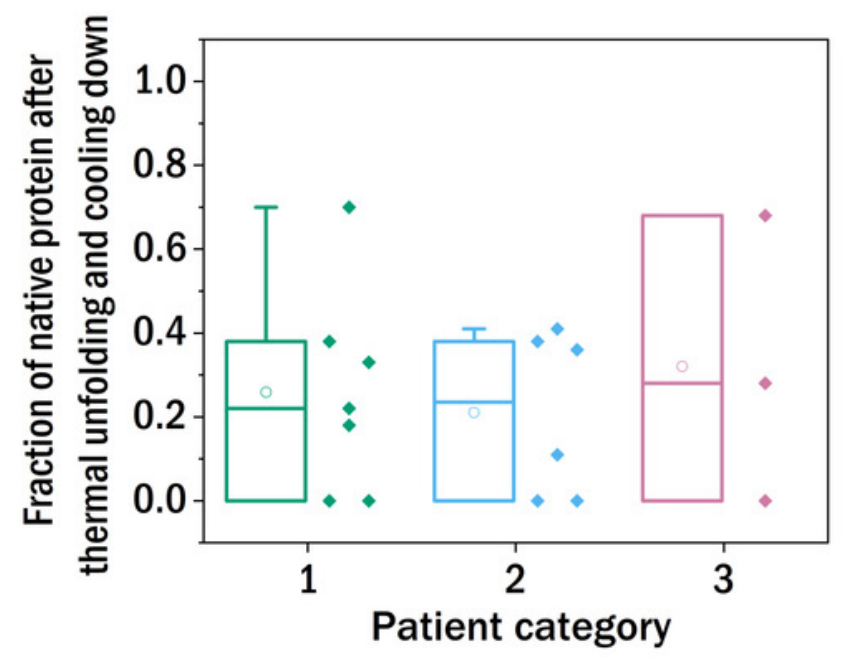


Figure 2

Monomer-dimer distribution of the LCs.

The relative fraction of dimer of the different samples measured in relation to the overall amount of native light chains (monomer and dimer), as determined by SDS-PAGE. The colors refer to the corresponding patient category defined above: I (green), II (blue), III (red), the shape indicates the isotype of the light chain: triangle: lambda, circles: kappa.

A

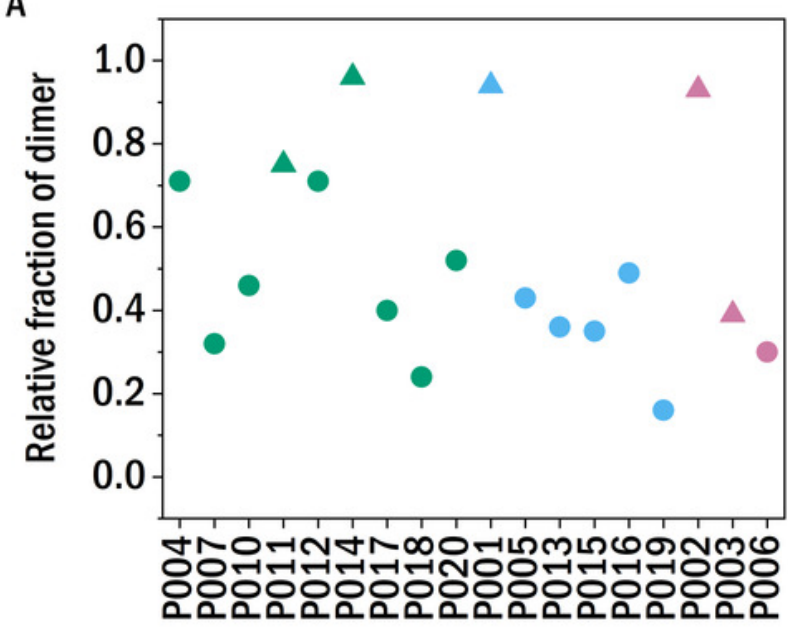

B

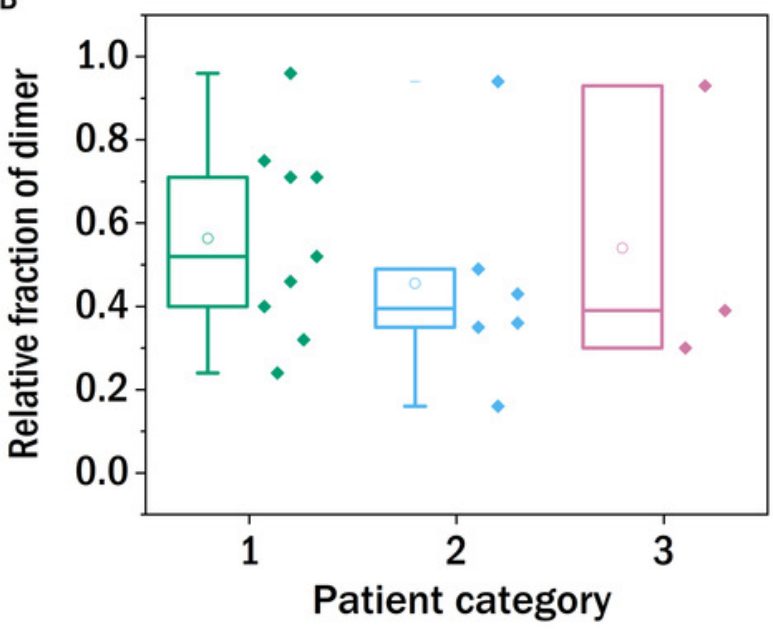




\section{Figure 3}

Trypsin digestion.

(A) \& (B) The fraction of native protein (monomer and dimer combined) after $48 \mathrm{~h}$ incubation with trypsin is displayed. The colors refer to the corresponding patient category defined above: I (green), II (blue), III (red). The shape of the symbols represents the isotype of the LC: triangles: lambda isotype, circles: kappa isotype. Empty circles: amyloid fibril formation induced by proteolysis and inferred from an increased signal of ThT-fluorescence, filled circles: no evidence for amyloid formation observed. (C) SDS-PAGE gel of the trypsin digestion of P013 as a representative example. The dimer (orange) and the monomer (purple) are marked with a square. (D) ThT fluorescence aggregation assay of the amyloid forming LCs. Data for P006 is added as an example of a LC that does not from amyloid fibrils. (E) AFM-image of the aggregated sample P007 after digestion with trypsin during the kinetic experiment in a multiwell plate. 
A

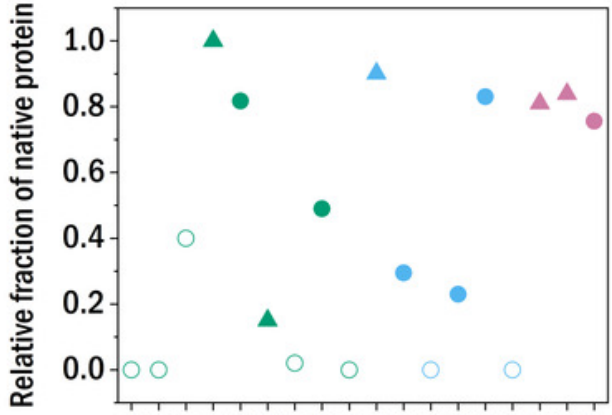

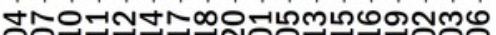
ริํำ
C

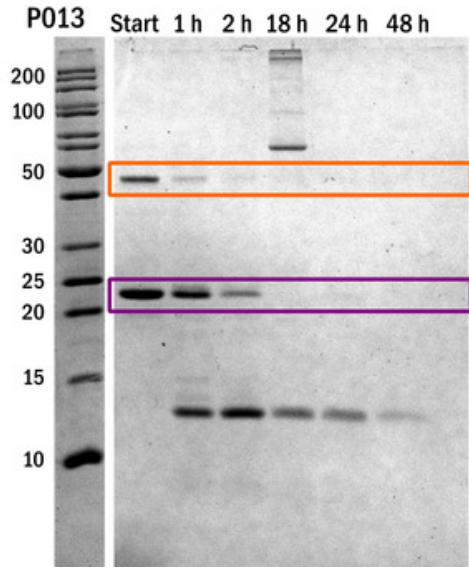

D

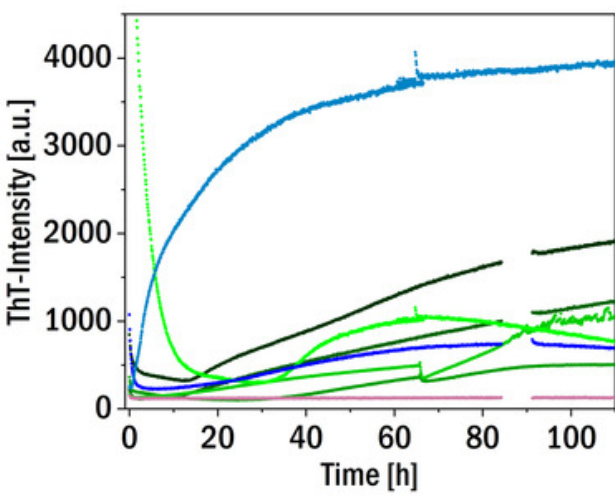

B

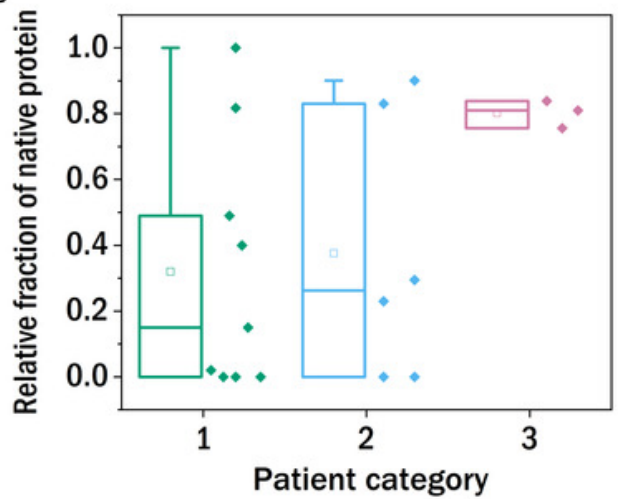

E

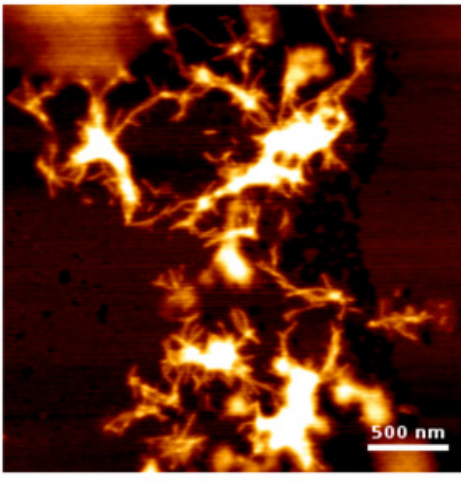

$11.3 \mathrm{~nm}$

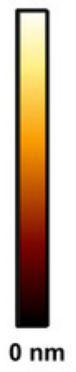




\section{Figure 4}

Amyloid fibril formation by P005 under different solution conditions as a representative example.

(A) Aggregation assay at different $\mathrm{pH}$ values in the presence of $1 \mathrm{mM}$ TCEP and (B) in the absence of TCEP. (C) Aggregation assay at $\mathrm{pH} 4$ at different monomer concentrations measured through ThT-fluorescence. (D) and (E) AFM images of the sample P005 at $50 \mu \mathrm{M}$, at the end of the experiment shown in (C). (F) The amyloid fibril formation behavior at $\mathrm{pH} 4$ of the LCs monitored by an increase in the fluorescence intensity of the dye ThT: yes: if the amyloid formation was observed; no: no evidence of amyloid fibril formation was observed. The colors refer to the corresponding patient category defined above: I (green), II (blue), III (red). The shape typify the isotype of the LC: triangles: lambda isotype, circles: kappa isotype.

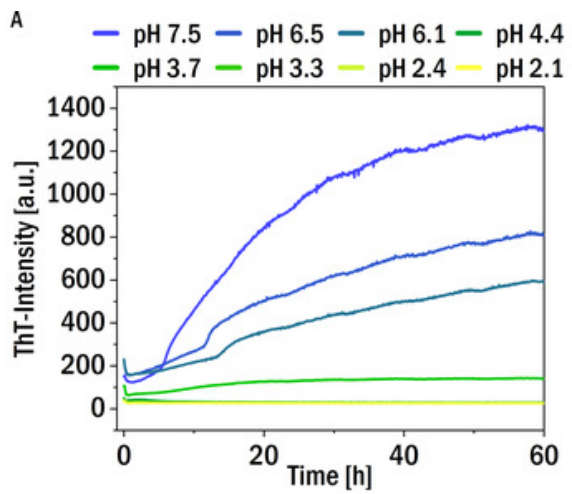

D

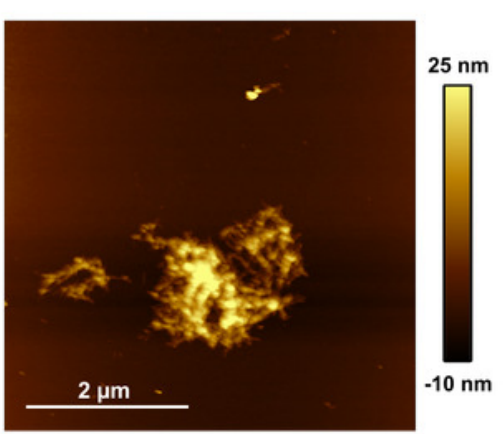

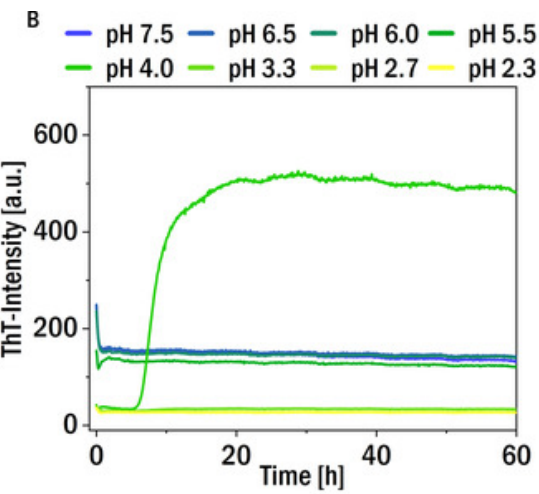

E

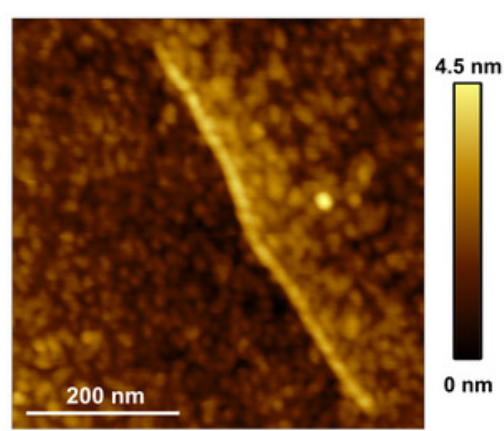

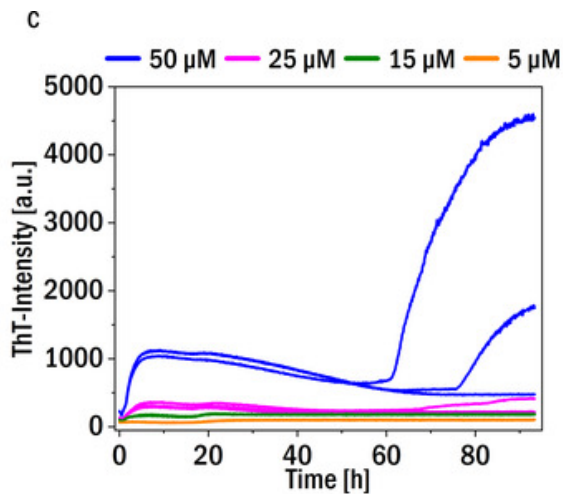

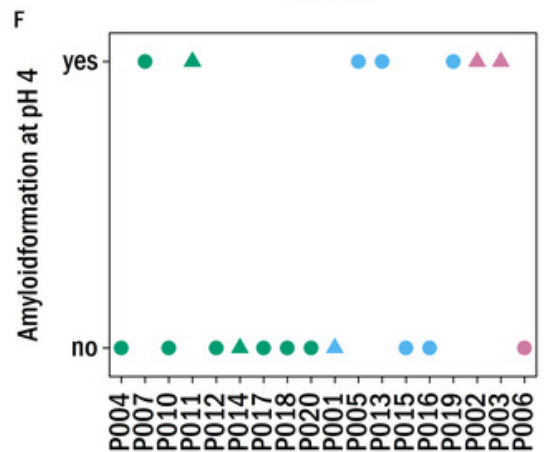


Figure 5

The Pearson correlation coefficient between the clinical patient categories and the different investigated biochemical and biophysical characteristics.

The points are labeled with the corresponding p-value.

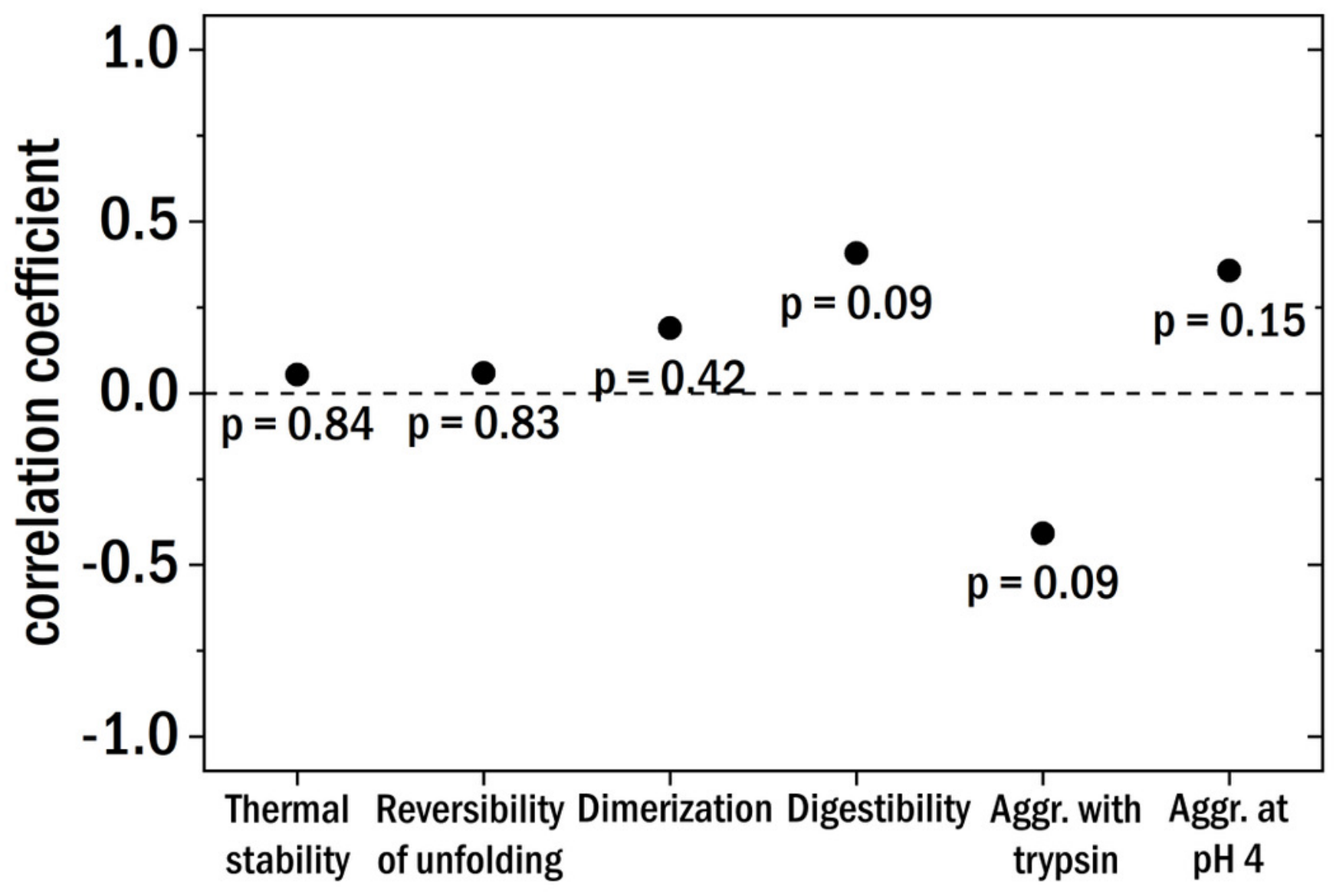




\section{Table $\mathbf{1}$ (on next page)}

Patient Characteristics at the time of examination.

The patient groups are given according to CKD: Group I good Stage 1+2 n $=9$ (P004, 007, 010, 011, 012, 014, 017, 018, 020), Group II intermediate Stage $3 \mathrm{n}=6$ (P001, 005, 013, $015,016,019)$ and Group III bad Stage $4+5 \mathrm{n}=5(\mathrm{P} 002,003,006,008,009)$ 
1 Table 1:

2 Patient Characteristics at the time of examination.

3 The patient groups are given according to CKD: Group I good Stage 1+2 n = 9 (P004, 007, 010, 011, 012, 014, 017, 018, 020), Group

4 II intermediate Stage $3 \mathrm{n}=6$ (P001, 005, 013, 015, 016, 019) and Group III bad Stage 4+5 n = $5(\mathrm{P} 002,003,006,008,009)$

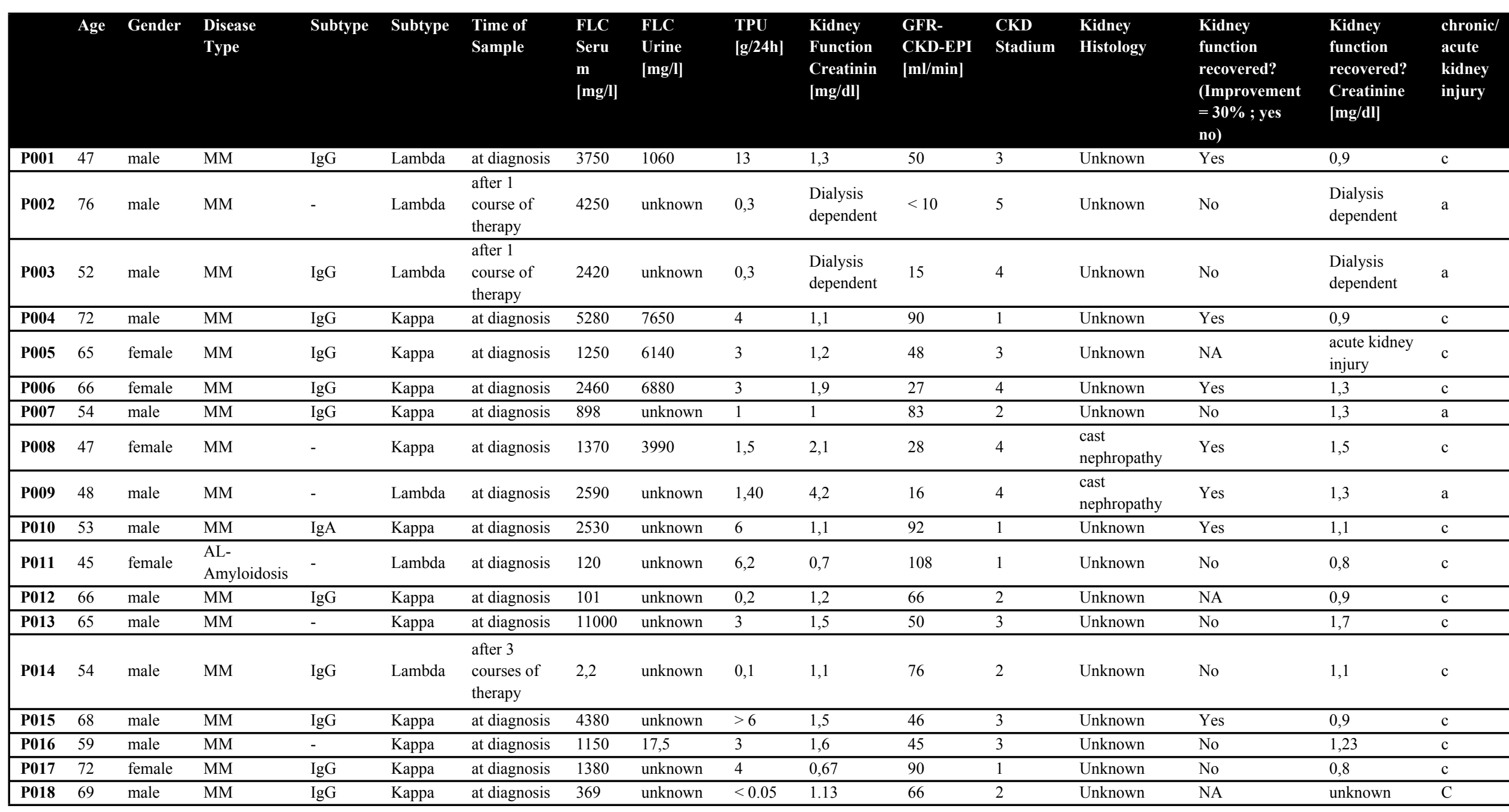




\begin{tabular}{|c|c|c|c|c|c|c|c|c|c|c|c|c|c|c|c|c|}
\hline P019 & 56 & male & $\mathrm{MM}$ & $\mathrm{IgG}$ & Kappa & at diagnosis & 484 & unknown & $<0.05$ & 1.79 & 41 & 3 & Unknown & Yes & 1.4 & $\mathrm{C}$ \\
\hline$\overline{\mathbf{P 0 2 0}}$ & 64 & female & $\overline{M M}$ & - & Kappa & at diagnosis & 4120 & unknown & 2.4 & 0.81 & 74 & 2 & Unknown & $\overline{\mathrm{NA}}$ & 0.85 & $\overline{\mathrm{C}}$ \\
\hline
\end{tabular}

